\title{
PROPUESTA DE INNOVACIÓN DOCENTE PARA LA ASIGNATURA: DE LA PLENITUD MEDIEVAL AL RENACIMIENTO
}

Silvia María Pérez González (spergon@upo.es)

Juan Carlos Arboleda Goldaracena (jcarbgol@upo.es)

Universidad Pablo de Olavide

Resumen: En el presente trabajo planteamos un conjunto de propuestas de innovación docente para el aprendizaje de una de las asignaturas asignadas al Área de Historia Medieval de la Universidad Pablo de Olavide, "De la Plenitud medieval al Renacimiento". Se formulan distintos modelos de trabajo para cada uno de los temas que conforman el temario, que incluye el trabajo en equipo y los foros de discusión para el comentario de textos históricos, películas, novelas, visitas. Todo ello basado en el empleo de la plataforma virtual. Finalmente, cada elemento susceptible de evaluación cuenta con la rúbrica correspondiente a fin de facilitar al alumno los criterios evaluables y los requisitos que han de cumplir. En definitiva, se trata a la vez de reflexionar sobre cómo los docentes estamos plasmando en la práctica distintas iniciativas de innovación, con especial atención a nuestro aprendizaje y visiones del alumnado. Al mismo tiempo queremos que el trabajo sea un reflejo de las diferentes maneras de abordar la innovación en el aula.

\begin{abstract}
The aim of this work is a set of teaching innovation proposals for the learning of one of the subjects assigned to the Area of Medieval History of the Pablo de Olavide University, "From the medieval Plenitude to the Renaissance". Different work models are formulated for each of the topics that make up the agenda, which includes teamwork and discussion forums for the commentary of historical texts, films, novels, and visits. All this based on the use of the virtual platform. Finally, each element subject to evaluation has the corresponding rubric in order to provide the student with the evaluable criteria and the requirements that must be met. In short, it is at the same time to reflect on how each of us is putting into practice our process of teacher training and innovation, with special attention to our learning and visions of the students. At the same time we want the work to be a reflection of the different ways of approaching innovation in the classroom.
\end{abstract}

Palabras clave: Edad Media. Nuevas tecnologías docentes. Aplicaciones en el aula. Materiales educativos. Modelos de trabajo. Docente. Estudiante universitario. Planificación de la educación. Innovación educativa. Tecnologías de la información.

Keywords: Middle Ages. New teaching technologies. Applications in the classroom. Educational materials. Work models. Teacher. College student. Education planning. Educational innovation. Information technology. 


\section{Introducción: Contextualización de la asignatura}

La asignatura "De la Plenitud Medieval al Renacimiento" es una asignatura obligatoria que se imparte en el primer cuatrimestre del grado de Humanidades y doble grado de Humanidades y Traducción e Interpretación. Tiene asignado un total de 7.5 créditos y se encuentra englobada en el módulo de Fundamentos Históricos. Desde el decanato de Humanidades se nos indica el porcentaje que hemos de dedicar a cada tipo de enseñanza: 50\% enseñanzas básicas, 40\% enseñanzas prácticas y de desarrollo y 10\% actividades dirigidas.

Varios son los condicionantes que hemos de tener en cuenta a la hora de diseñar la planificación (Gil Flores, 2004, Bernal, 2006) de la asignatura en parámetros de innovación docente. En primer lugar es necesario considerar que esta asignatura es continuación en el segundo curso del grado de Humanidades y el doble grado de Humanidades y Traducción e Interpretación de la impartida en el primer curso que lleva por título "La conformación de las civilizaciones cristiana e islámica medievales”. Por tanto, el alumno habrá de recibir, a través de las distintas vías que se implementarán, la formación necesaria para afrontar sin problemas la continuación de la asignatura recibida en el curso anterior (Morales, 2005).

Por otro lado, dado que en el nuevo contexto surgido al amparo del Espacio Europeo de Educación Superior el alumno (Del Val, 2013) se convierte en protagonista decisivo de su proceso de aprendizaje, será necesario considerar y reflexionar sobre el grupo de estudiantes al que la asignatura se va a impartir. La Facultad de Humanidades ofrece 30 plazas para los alumnos de Humanidades y otras 30 para los que van a cursar el doble grado. Ello suma un total de 60 alumnos. En principio el número puede limitar la aplicación de determinadas innovaciones docentes. Pero hemos de subrayar que la parte práctica de la asignatura se desglosa en dos grupos de tal manera que, aunque con motivo de las enseñanzas básicas (Danvila, 2013) contemos con 60 alumnos en el aula, en lo que se refiere a enseñanzas prácticas y de desarrollo, actividades dirigidas, tutorías, visitas académicas, correcciones, solo hemos de responsabilizarnos de la mitad, 30, un número muy adecuado para introducir las más variadas innovaciones docentes.

Pero en relación con los alumnos no solo hemos de tener en cuenta el número, sino también otros factores como la edad del colectivo estudiantil para el que vamos a diseñar la asignatura. La media de edad de los estudiantes es de unos 19-20 años, que ya en el primer curso han empezado a experimentar los nuevos caminos de la docencia. No obstante en los últimos años estamos asistiendo a una interesante llegada a las aulas de alumnos mayores de 25 años. Hablamos de interesante llegada porque muchas veces ayudan al profesor en las carencias de maduración que presentan los alumnos recién ingresados tras las pruebas de acceso a la 
Universidad. Por supuesto que estos alumnos “mayores", muchos de ellos ya diplomados o licenciados que están trabajando y que acuden a la Facultad de Humanidades con el fin de ampliar sus estudios y su preparación, tienen unos intereses y motivaciones distintas a las de sus compañeros. Pero en general se adaptan bien a los ritmos y pautas que el Espacio Europeo de Educación Superior (Metcalfe, 2006) les exige.

Antes de pasar a los siguientes apartados queremos indicar que muchos de esos ritmos y pautas forman parte de la guía docente que los alumnos tienen a su disposición a comienzos de la asignatura.

\section{Objetivos}

El objetivo teórico de esta asignatura es el estudio del pasado humano en sus diversos aspectos durante la Plena y Baja Edad Media, circunscrita al ámbito europeo-mediterráneo, con especial énfasis en la Europa cristiana como cuna de un determinado concepto de Europa vigente hasta nuestros días y principal conformador de la realidad continental hasta, al menos, el siglo XIX. El estudio de esta época permite al alumno conocer los grandes procesos históricos que construyeron los cimientos del período medieval durante el cual coexistieron tres grandes civilizaciones en el ámbito mediterráneo: el imperio de Oriente o Bizancio, el Islam y la Cristiandad latina. Al mismo tiempo se le introduce en un método que potencia la síntesis explicativa, basado en la capacidad de seleccionar, jerarquizar y articular los acontecimientos de la Historia Medieval en los procesos históricos de los que forman parte. Finalmente, no debemos olvidar que la Edad Media constituye una etapa fundamental de nuestro pasado histórico, que tan profundas huellas tiene en el presente.

A este objetivo (que sobre todo hace referencia a competencias cognitivas) queremos añadir otros que la asignatura nos permite diseñar al hilo de sus contenidos teóricos. Nuestra finalidad no es formar alumnos expertos en Historia Medieval, que lo sepan todo sobre este periodo y sus protagonistas y procesos. Nuestro deseo es que a través de esta asignatura adquieran las herramientas para encontrar toda la información que nosotros no les vamos a proporcionar, que aprendan a analizarla desde la crítica y el método histórico, que sean capaces de separar los contenidos válidos de los infructuosos y, también muy importante, que se desarrolle en ellos el sentido crítico a la hora de analizar el presente como momento histórico susceptible de ser transformado. En definitiva, y esperamos no caer en la utopía (Nevot, 2008), hacerles ver a través del pasado medieval que no todo lo que se les ofrece es válido y verdadero, sino que detrás de ello se pueden ocultar otras realidades e intenciones.

(c) B EY-NC-ND 2018, Universitat Politècnica de València Congreso In-Red (2018) 
Para ello analizaremos textos históricos que desde distintas perspectivas aborden un mismo acontecimiento, les enseñaremos los instrumentos del método histórico, organizaremos visitas a distintos monumentos para ver in situ su función política, social, económica y, si es el caso, religiosa, en la sociedad que los generó, escogiéndose los materiales de trabajo de acuerdo con los objetivos planteados.

\section{Desarrollo de la innovación}

\subsection{Competencias}

Este apartado constituye una de las grandes innovaciones en términos docentes que ha experimentado nuestra docencia. La actual enseñanza universitaria se concibe en términos competenciales, siendo necesario tanto por parte del profesor (Knight, 2005) como del alumno conocer las competencias que se van a trabajar y la metodología que se les va a aplicar (Miguel Díaz, 2006).

Para la selección de las competencias que se van a trabajar en la asignatura "De la Plenitud Medieval al Renacimiento" hemos tenido muy en cuenta los perfiles profesionales recogidos en la Memoria de verificación del título de graduado en Humanidades por la Universidad Pablo de Olavide. Finalmente nos hemos decidido a trabajar aquellas competencias que sean comunes a todos ellos, para que a la hora de enfrentarse a su futuro profesional los alumnos cuenten con instrumentos que aplicar a todas las salidas profesionales. También hemos tenido en cuenta el periodo temporal de que disponemos para el trabajo competencial, lo que nos ha llevado a seleccionar las tres siguientes:

\section{a) Instrumental:}

Comunicarse con corrección en lengua española, oralmente y por escrito, con un alto nivel en el uso de los diferentes recursos expresivos y con dominio del registro académico y científico.

b) Actitudinal:

Saber trabajar en un equipo en un equipo de forma cooperativa y responsable, respetando los principios de igualdad y diversidad como es propio de una cultura democrática, y buscando el entendimiento para la consecución de objetivos.

c) Sistémica:

Desarrollar la capacidad de aprendizaje autónomo.

\subsection{Contenidos de la asignatura (Temario)}

Lección 1: Aspectos generales del desarrollo económico y social del Occidente europeo durante la Plena Edad Media

(c)) EY-NC-ND 2018, Universitat Politècnica de València

Congreso In-Red (2018) 
I. Vínculos sociales plenomedievales

1. Introducción.

I. Vínculos horizontales. El sistema feudal. 3. Vínculos verticales. El señorío plenomedieval. IIDemografía. III- Economía rural. IV-Renacimiento urbano. IV- Economía urbana: Artesanía y comercio

Lección 2: El pontificado y la Reforma de la Iglesia

1. Clero Regular. 1.a) El monacato. 1.b) Las Órdenes Mendicantes.2-.Clero secular

Lección 3: Del renacimiento del siglo XII al apogeo de la cultura medieval

1. El paso de la escuela catedralicia a la universidad. 2. El nacimiento de las Universidades en el siglo XIII. 2.a) La gran triada universitaria europea del siglo XIII. 3. Organización universitaria. 4. La enseñanza. 4.a) Los estudios. 4.b) Los exámenes, 4.c) Calendario lectivo. 4.d) Los libros. 4.e) El método escolástico

Lección 4: Las estructuras políticas en la plenitud medieval

I. Las Cruzadas. 1. Los escenarios de las Cruzadas. 2. Causas de las Cruzadas. 2.a) Causas materiales. 2.b) Causas mentales. 3. La primera Cruzada (1097-1099). 4. La segunda Cruzada (1147-1149). 5. La tercera Cruzada (1189-1190). 6. La cuarta Cruzada (1202-1204). 7. La Cruzada infantil (1212). 8. La quinta Cruzada (1228-1229). 9. La sexta Cruzada (1248-1254). 10. La séptima Cruzada (1270). 11. Consecuencias de las Cruzadas. II. Las monarquías feudales

Lección 5: La Baja Edad Media ¿Tiempo de crisis?. La Iglesia. Las dificultades del pontificado y la religión como subversión

1. Introducción. 2. Periodización de la crisis. 3. Factores de la crisis. 3.a) El hambre. 3.b) La guerra. 3.c) La peste. 4. Consecuencias en el ámbito rural: Tensiones y luchas sociales. 4. a) en Flandes. 4. b) en Francia. 4.c) en Inglaterra. 5. La crisis en las ciudades: Las revueltas sociales en el medio urbano. 5.a) En Italia. 5.b) en Francia. 6. La Iglesia bajomedieval. 6.a) El pontificado de Aviñón (1309-1377). 6.b) El cisma de Occidente. 6.c) Situación de la Iglesia a fines del siglo XV. 7. Las transformaciones de la vida espiritual: los nuevos caminos de la piedad y las herejías. 7.a) Devotio moderna. 7.b) la piedad popular. 7.c) Las herejías.

En la siguiente matriz de especificaciones se indica la relevancia que cada tema tiene dentro de la asignatura:

\begin{tabular}{|c|c|c|c|c|c|}
\hline \multirow[b]{2}{*}{$\begin{array}{c}\text { Unidad de } \\
\text { competencia/Temas }\end{array}$} & \multirow[b]{2}{*}{$\begin{array}{c}\text { Pesos } \\
\text { pondera- } \\
\text { tivos }\end{array}$} & \multirow[b]{2}{*}{$\begin{array}{c}\mathrm{N}^{\mathbf{o}} \\
\text { ítems }\end{array}$} & \multicolumn{3}{|c|}{$\begin{array}{c}\text { Prueba de conocimientos }\left(\mathrm{N}^{\circ} \text { ítems y por niveles }\right. \\
\text { taxonómicos) }\end{array}$} \\
\hline & & & $\begin{array}{c}\text { Conoci- } \\
\text { mientos } \\
\left(\mathbf{N}^{0} \text { ítems) }\right.\end{array}$ & $\begin{array}{l}\text { Comprensión } \\
\text { (No ítems) }\end{array}$ & $\begin{array}{l}\text { Aplicación } \\
\text { (No ítems) }\end{array}$ \\
\hline $\begin{array}{l}\text { 1. Aspectos generales } \\
\text { socio-económicos }\end{array}$ & 10 & 3 & 2 & 1 & \\
\hline
\end{tabular}

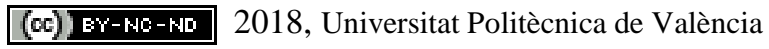

Congreso In-Red (2018) 


\begin{tabular}{|l|c|c|c|c|c|}
\hline $\begin{array}{l}\text { 2. El Pontificado y la } \\
\text { refoema de la Iglesia }\end{array}$ & 20 & 7 & 2 & 5 & \\
\hline $\begin{array}{l}\text { 3. Del apogeo al } \\
\text { renacimiento cultural }\end{array}$ & 25 & 15 & 4 & 8 & 4 \\
\hline $\begin{array}{l}\text { 4. Las estructuras políticas } \\
\text { plenomedievales }\end{array}$ & 25 & 15 & 4 & 7 & 6 \\
\hline 5. La Baja Edad Media & 20 & 10 & & 4 & 13 \\
\hline TOTAL & 100 & 50 & 12 & 25 & 4 \\
\hline
\end{tabular}

\subsection{Metodología y recursos}

Cada tema tendrá su propia metodología dentro del proceso de aprendizaje, que pasamos a especificar.

Tema 1: dentro de las clases correspondientes a las Enseñanzas prácticas y de desarrollo se analizarán los vínculos horizontales, concretamente el sistema feudal. En el apartado de los vínculos verticales estudiaremos el señorío plenomedieval, dividido en territorial y jurisdiccional. Los siguientes bloques se corresponden con II. Demografía, III. Economía rural, IV.Renacimiento urbano, V. Economía urbana: Artesanía y VI. Economía urbana: Comercio. Lo hacemos a través distintas fuentes históricas: documentos contemporáneos al proceso, visto por autores que estaban a favor de un hecho y en contra, y documentos de épocas posteriores donde los elementos medievales sirven como fundamento a creaciones políticas muy posteriores. Estos textos se comentan en las clases (Rivero, 2003) y también son objeto de debates en el foro del aula virtual (Hanna, 2002), herramienta a través de la cual les son proporcionados los textos. Para todo ello los alumnos se organizan en equipos (Elboj, 2002) que han de defender distintas posturas que ellos mismos eligen. A título individual cada estudiante debe elegir una película (v.g. El mercader de Venecia) sobre el periodo estudiado que habrá de comentar brevemente en las clases dedicadas a las actividades dirigidas.

Dado que se trata del primer tema de la asignatura consideramos de gran importancia introducir una sesión de tutoría (Álvarez, 2008), que es la primera de las programadas, con el objetivo de cumplir el nuevo papel que se le asigna al profesor dentro del marco de enseñanza y de los criterios establecidos en la Convención de Bolonia y en la legislación española adaptada al Espacio Europeo de Educación Superior. El docente debe adoptar la posición de tutor, que acompañará, seguirá y apoyará al estudiante en su proceso de crecimiento personal y aprendizaje profesional (Medina, 2003). En esta primera tutoría se trata de orientar al alumno en cuantas cuestiones considere que pueden ser importantes, en los problemas de comprensión y análisis que los textos puedan generarle, dejando siempre que el estudiante plantee soluciones y herramientas que puedan serle útiles en la resolución de los obstáculos que se le hayan podido 
plantear. Con ello queremos potenciar en el alumno la capacidad de desarrollar un aprendizaje continuo a lo largo de la vida (McIntosh, 2005), de tener un papel mucho más activo en la construcción de su propio aprendizaje. Atrás deben quedar esas tutorías destinadas tan solo a resolver dudas antes del examen; se trata de asistir al estudiante en la ejecución de su proyecto tanto personal como profesional.

De esta tutoría así como de las siguientes llevamos a cabo un registro en el que recogemos toda la información (observaciones, reflexiones, datos concretos, etc.) que consideremos necesarios para atender y tutorizar a los alumnos en los procesos de enseñanzaaprendizaje. Se trata de cuestiones como: preguntas planteadas por los alumnos o dudas que les surjan y respuestas a las mismas aportadas por ellos o por el profesor; cuestiones problemáticas en temas complejos; dificultades de los materiales que se han propuesto para ser trabajados; implicación e interés del alumno en las investigaciones que se le propone; dificultades surgidas en la tutoría; tiempo de desarrollo de la tutoría; idoneidad de la tutoría celebrada.

Tema 2: los contenidos teóricos se acompañan de la lectura de algunos capítulos seleccionados de la Vida de San Francisco y sobre la novela Imperator (Velasco, 2014). Ambas monografías son objeto de análisis por parte de los alumnos quienes por equipos, cuyos miembros deberán ser diferentes a los de los grupos constituidos para el tema anterior a fin de conseguir que todos trabajen con todos, deben exponer qué parte de ambos discursos pueden tener su aplicación a la contemporaneidad y en qué medida unos mismos procesos históricos pueden desarrollarse en momentos diferentes de la Historia, aun cuando los protagonistas, artífices y circunstancias sean otros. A nivel autónomo (Alonso, 2007) cada estudiante deberá buscar en los medios de comunicación esa huella del pasado de la Iglesia medieval. Preparamos también un blog con fotos de los conventos y monasterios de los pueblos originarios de los alumnos, donde además de la foto deberán incluir un comentario de su experiencia del contacto con un pasado que no deja de ser presente.

Una vez impartidos estos dos primeros temas el alumno ya estará en condiciones de asistir a una tutoría en la que se trabajará la competencia instrumental relativa a la comunicación correcta en lengua española, oralmente y por escrito, con un alto nivel en el uso de los diferentes recursos expresivos y con dominio del registro académico y científico. Esta tiene lugar durante el segundo mes de impartición de la asignatura y durante la misma se pregunta al estudiante sobre cuestiones relativas a estos dos primeros temas y se le pide que realice por escrito una síntesis muy breve de su opinión acerca del desarrollo de los mismos. Para la evaluación (Rodríguez de Miñón, 2003) de la competencia indicada introducimos la siguiente rúbrica (Fernández, 2016): 
Tabla 1: Rúbrica de evaluación de la competencia: comunicación escrita en lengua propia ${ }^{1}$

\begin{tabular}{|c|c|c|c|c|}
\hline & CORRECTA & BUENA & EXCELENTE & PUNTOS \\
\hline \multicolumn{5}{|l|}{ Lengua escrita } \\
\hline Expresión escrita & \begin{tabular}{|ll|}
\multicolumn{3}{|c|}{ Siguen un orden y } \\
construyen las frases \\
correctamente
\end{tabular} & $\begin{array}{|llr|}\text { Siguen un } & \text { orden y construyen las } \\
\text { frases } & \text { correctamente. } & \mathrm{El} \\
\text { vocabulario es adecuado } & \end{array}$ & $\mid \begin{array}{l}\text { Siguen un orden y construyen las frases } \\
\text { correctamente. El vocabulario es rico y la } \\
\text { lectura muy agradable }\end{array}$ & \\
\hline \multirow{2}{*}{$\begin{array}{l}\text { Ortografía } \\
\text { gramática }\end{array}$} & \begin{tabular}{|l|} 
Hay algunos errores de \\
sintaxis, ortografía o léxico
\end{tabular} & Hay algunos errores de sintaxis. & \begin{tabular}{|l} 
No hay prácticamente ningún error de \\
ningún tipo.
\end{tabular} & \\
\hline & $1-2-3$ & $4-5-6$ & $7-8-9$ & \\
\hline \multirow[t]{2}{*}{$\begin{array}{l}\text { Organización del } \\
\text { trabajo }\end{array}$} & 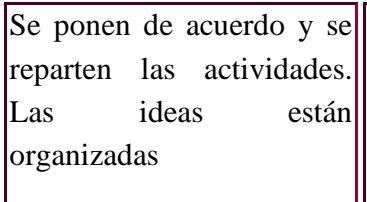 & \begin{tabular}{|l} 
Se ponen de acuerdo y se reparten \\
las actividades. Las ideas están \\
organizadas y complementadas con \\
ejemplos
\end{tabular} & $\mid \begin{array}{l}\text { Se ponen de acuerdo y se reparten las } \\
\text { actividades. Las ideas están perfectamente } \\
\text { organizadas, complementadas con detalles } \\
\text { y explicaciones aclaratorias }\end{array}$ & \\
\hline & $1-2-3$ & $4-5-6$ & $7-8-9$ & \\
\hline El resumen & Incluyen un resumen & \begin{tabular}{|l} 
Incluyen un resumen que cierra \\
correctamente el tema
\end{tabular} & $\begin{array}{l}\text { Incluyen un resumen que retoma las ideas } \\
\text { principales y cierra el tema de forma } \\
\text { especialmente atractiva. }\end{array}$ & \\
\hline TOTALES & & & & \\
\hline
\end{tabular}

Tema 3: se proyecta la película El nombre de la rosa, para que los alumnos identifiquen los elementos de la Iglesia medieval que incluye el film, siendo voluntario consultar la novela (Lobatón, 2013) homónima. Tras la explicación del tema se realiza una visita a Jerez de la Frontera para que los alumnos comprueben in situ el paso de la civilización islámica a la civilización cristiana. Los distintos hitos arquitectónicos son explicados por nuevos equipos de alumnos que eligen de forma autónoma el elemento que van a exponer. En una nueva sesión de tutoría han de tratar con el profesor los problemas que les han surgido en la preparación de la exposición, los motivos de la elección del tema, los recursos manejados, etc. Esta tutoría es un buen momento para evaluar la competencia actitudinal relativa al trabajo en grupo mediante la siguiente rúbrica:

\footnotetext{
${ }^{1}$ Tomada de: http://www.juntadeandalucia.es/averroes/cepmotril/webquest/trabajos/4esta/evaluacion.doc
} 
Tabla 2. Rúbrica de evaluación de la competencia: saber trabajar en equipo ${ }^{2}$

\begin{tabular}{|c|c|c|c|c|c|}
\hline Descriptores & 1 & 2 & 3 & 4 & 5 \\
\hline \multirow[t]{2}{*}{$\begin{array}{l}\text { Indicador 1: } \\
\text { Realiza las tareas } \\
\text { que le son } \\
\text { asignadas por el } \\
\text { grupo dentro de } \\
\text { los plazos } \\
\text { requeridos }\end{array}$} & $\begin{array}{l}\text { No cumple las tareas } \\
\text { asignadas }\end{array}$ & $\begin{array}{l}\text { Cumple } \\
\text { parcialmente las } \\
\text { tareas asignadas } \\
\text { y/o no cumple } \\
\text { con los plazos } \\
\text { requeridos }\end{array}$ & $\begin{array}{l}\text { Cumple las } \\
\text { tareas } \\
\text { asignadas en los } \\
\text { plazos requeridos }\end{array}$ & $\begin{array}{l}\text { Cumple las tareas } \\
\text { asignadas en los } \\
\text { plazos requeridos } \\
\text { y la calidad de la } \\
\text { tarea supone un } \\
\text { notable aporte al } \\
\text { equipo }\end{array}$ & $\begin{array}{l}\text { Cumple las tareas } \\
\text { asignadas en los } \\
\text { plazos requeridos y la } \\
\text { calidad de la tarea } \\
\text { supone un notable } \\
\text { aporte al } \\
\text { equipo. }\end{array}$ \\
\hline & 1 & 2 & 3 & 4 & 5 \\
\hline \multirow[t]{2}{*}{$\begin{array}{l}\text { Indicador 2: } \\
\text { Participa de } \\
\text { forma activa en } \\
\text { los espacios de } \\
\text { encuentro del } \\
\text { equipo, } \\
\text { compartiendo la } \\
\text { información, los } \\
\text { conocimientos y } \\
\text { las experiencias }\end{array}$} & $\begin{array}{l}\text { Se ausenta con } \\
\text { frecuencia y su } \\
\text { presencia } \\
\text { irrelevante }\end{array}$ & $\begin{array}{l}\text { Interviene poco } \\
\text { en el debate, } \\
\text { principalmente a } \\
\text { requerimiento de } \\
\text { los } \\
\text { demás }\end{array}$ & $\begin{array}{lr}\text { Se muestra } \\
\text { activo } \\
\text { participativo en } \\
\text { los encuentros de } \\
\text { grupo }\end{array}$ & $\begin{array}{l}\text { Se muestra activo } \\
\text { y participativo en } \\
\text { los encuentros de } \\
\text { grupo. Con sus } \\
\text { intervenciones } \\
\text { fomenta } \\
\text { participación y } \\
\text { mejora la calidad } \\
\text { de los resultados } \\
\text { del equipo }\end{array}$ & $\begin{array}{l}\text { Se muestra activo y } \\
\text { participativo en los } \\
\text { encuentros de grupo. } \\
\text { Con sus } \\
\text { intervenciones } \\
\text { fomenta } \\
\text { participación y } \\
\text { mejora la calidad de } \\
\text { los resultados del } \\
\text { equipo. Sus } \\
\text { contribuciones son } \\
\text { fundamentales tanto } \\
\text { para el proceso } \\
\text { grupal como para la } \\
\text { calidad del resultado }\end{array}$ \\
\hline & 1 & 2 & 3 & 4 & 5 \\
\hline \multirow[t]{2}{*}{$\begin{array}{l}\text { Indicador 3: } \\
\text { Colabora en la } \\
\text { definición, } \\
\text { organización y } \\
\text { distribución } \\
\text { de las tareas de } \\
\text { grupo }\end{array}$} & $\begin{array}{l}\text { Manifiesta } \\
\text { resistencias } \\
\text { ante la organización } \\
\text { del } \\
\text { trabajo en el equipo }\end{array}$ & $\begin{array}{lr}\text { Se limita } & \text { a } \\
\text { aceptar } & \text { la } \\
\text { organización } & \text { del } \\
\text { trabajo propuesta } \\
\text { por r } \\
\text { miembros } & \text { otros } \\
\text { equipo } & \text { del } \\
\end{array}$ & $\begin{array}{l}\text { Participa en la } \\
\text { planificación, } \\
\text { organización } \\
\text { y distribución del } \\
\text { trabajo en } \\
\text { equipo }\end{array}$ & $\begin{array}{l}\text { Participa en la } \\
\text { planificación, } \\
\text { organización } \\
\text { y distribución del } \\
\text { trabajo en } \\
\text { equipo. } \\
\text { organizado } \\
\text { distribuye } \\
\text { las tareas con } \\
\text { eficacia }\end{array}$ & $\begin{array}{l}\text { Participa en la } \\
\text { planificación, } \\
\text { organización } \\
\text { y distribución del } \\
\text { trabajo en equipo. Es } \\
\text { organizado } \\
\text { distribuye } \\
\text { las tareas con } \\
\text { eficacia. Fomenta } \\
\text { una organización del } \\
\text { trabajo aprovechando } \\
\text { los recursos de los } \\
\text { miembros del equipo }\end{array}$ \\
\hline & 1 & 2 & 3 & 4 & 5 \\
\hline $\begin{array}{l}\text { Indicador 4: } \\
\text { Toma en cuenta } \\
\text { los puntos de } \\
\text { vista de los } \\
\text { demás y } \\
\text { retroalimenta de }\end{array}$ & $\begin{array}{l}\text { No escucha las } \\
\text { intervenciones de sus } \\
\text { compañeros y las } \\
\text { descalifica } \\
\text { sistemáticamente. } \\
\text { Quiere imponer sus }\end{array}$ & $\begin{array}{l}\text { Escucha poco, no } \\
\text { pregunta, no se } \\
\text { preocupa por la } \\
\text { opinión de los } \\
\text { otros. Sus } \\
\text { intervenciones }\end{array}$ & $\begin{array}{l}\text { Acepta las } \\
\text { opiniones de los } \\
\text { otros y sabe dar } \\
\text { su punto de vista } \\
\text { en forma } \\
\text { constructiva }\end{array}$ & $\begin{array}{l}\text { Acepta las } \\
\text { opiniones de los } \\
\text { otros y sabe dar } \\
\text { su punto de vista } \\
\text { en forma } \\
\text { constructiva. }\end{array}$ & $\begin{array}{l}\text { Acepta las opiniones } \\
\text { de los otros y sabe } \\
\text { dar su punto de vista } \\
\text { en forma } \\
\text { constructiva. } \\
\text { Fomenta el diálogo }\end{array}$ \\
\hline
\end{tabular}

2 http://www.lapetus.uchile.cl/lapetus/recursos/documentos/Ejemplo_Rubrica_trabajo_en_equipo_UFGBE.pdf

(cc) EY-NC-ND 2018, Universitat Politècnica de València

Congreso In-Red (2018) 


\begin{tabular}{|c|c|c|c|c|}
\hline $\begin{array}{l}\text { forma } \\
\text { constructiva }\end{array}$ & opiniones & $\begin{array}{l}\text { son } \\
\text { redundantes y } \\
\text { poco sugerentes }\end{array}$ & $\begin{array}{l}\text { Fomenta } \\
\text { diálogo } \\
\text { constructivo } \\
\text { inspira } \\
\text { participación de } \\
\text { calidad de los } \\
\text { otros miembros } \\
\text { del grupo }\end{array}$ & $\begin{array}{l}\text { constructivo e inspira } \\
\text { la participación de } \\
\text { calidad de los otros. } \\
\text { Integra las opiniones } \\
\text { de los otros en una } \\
\text { perspectiva } \\
\text { superior, } \\
\text { manteniendo un } \\
\text { climar } \\
\text { colaboración y apoyo } \\
\text { a miembros del } \\
\text { grupo. }\end{array}$ \\
\hline
\end{tabular}

Tema 4: las clases teóricas se completan con un documental sobre el proceso político, económico, social y cultural que supusieron las Cruzadas. En colaboración con profesores del grado en Traducción e Interpretación, se ha puesto en práctica en algunos cursos una iniciativa consistente en que los alumnos explican a sus compañeros de la otra titulación los orígenes de la actual Francia, que precisamente se encuentran en el reinado de los Capetos que, junto al de los Angevinos, forma parte de nuestro temario, mientras que los alumnos de Traducción ilustran a los de Humanidades sobre la actual Francia. Fruto del intercambio de conocimientos (Gibbons, 1995) cada alumno, a nivel individual, realiza un pequeño trabajo sobre la huella del pasado capeto y angevino en la Francia de la contemporaneidad. Nuestras ambiciones van un poco más lejos y en un futuro pretendemos llevar a los dos grupos de alumnos a la Aquitania francesa, la antigua Aquisgrán, que fue la capital del Imperio de Carlomagno. El objetivo es contemplar in situ las huellas del pasado plenomedieval francés y su evolución posterior. Otro recurso es la proyección de películas como El Reino de los Cielos, Robin Hood o Templario para analizar las Cruzadas. La parte de Historia política del tema se trabaja a través de películas como La princesa prometida, la serie Los pilares de la tierra, o El león en invierno, aunque siempre aceptando propuestas de los propios alumnos. La metodología de trabajo de las películas es la ya comentada para otros materiales: identificación de los elementos propios del tema expuesto y explicación de los mismos.

Tema 5: la Baja Edad Media se explica a partir de distintos textos y manifestaciones varias, que son comentados en clases con un carácter eminentemente práctico. Los ejemplos ofrecidos por los profesores deben ser enriquecidos con otros por los alumnos. Se visualiza la película Paseo por el amor y la muerte. Los conocimientos adquiridos con motivo de esta lección son plasmados en un trabajo sobre "El uso actual de la Edad Media”. 


\subsection{Temporalización}

La asignatura se desarrolla a lo largo de 16 semanas, recibiendo el alumno cada semana dos horas de clases teóricas y una de clases prácticas. A estas horas se han de añadir las que el estudiante debe dedicar de forma autónoma o en equipo a las distintas actividades fuera del horario lectivo, así como las planificadas para las actividades dirigidas. El cronograma para un posible curso en el que se incorporarn todas las iniciativas propuestas es el siguiente:

Tabla 3. Cronograma

\begin{tabular}{|c|c|c|c|}
\hline SEMANA & $\begin{array}{c}\text { TEMA } \\
\text { TEÓRICO }\end{array}$ & PRÁCTICAS & TUTORÍA \\
\hline 1 & 1 & Comentario de textos & \\
\hline 2 & 1 & Comentario de textos y películas & \\
\hline 3 & 2 & Comentario de textos & $\mathrm{X}$ \\
\hline 4 & 2 & Comentario de textos & \\
\hline 5 & 2 & Comentario de textos & $\mathrm{X}$ \\
\hline 6 & 3 & Comentario de textos & \\
\hline 7 & 3 & Organización de la visita & $\mathrm{X}$ \\
\hline 8 & 3 & Organización de la visita & \\
\hline 9 & 3 & Visita & \\
\hline 10 & 4 & Documental & \\
\hline 11 & 4 & $\begin{array}{l}\text { Intercambio de conocimientos con los } \\
\text { alumnos de Traducción }\end{array}$ & \\
\hline 12 & 4 & $\begin{array}{l}\text { Intercambio de conocimientos con los } \\
\text { alumnos de Traducción }\end{array}$ & \\
\hline 13 & 4 & ¿Visita a Aquisgrán? & \\
\hline 14 & 5 & $\begin{array}{lccc}\text { Comentario de } & \text { textos } & \text { y } \\
\text { manifestaciones varias } & & \end{array}$ & \\
\hline 15 & 5 & $\begin{array}{lcc}\text { Comentario de } & \text { textos } \quad y \\
\text { manifestaciones varias } & & \end{array}$ & \\
\hline 16 & 5 & $\begin{array}{lccc}\text { Comentario de } & \text { textos } & \text { y } \\
\text { manifestaciones varias } & & \end{array}$ & \\
\hline
\end{tabular}

\section{Resultados: la evaluación}

La evaluación de los alumnos se realiza a través de distintas herramientas. Una de ellas es la realización de un portafolios. En el mismo se recogen los distintos trabajos que el estudiante ha llevado a cabo a lo largo del curso. Aunque la entrega definitiva del mismo tiene lugar al final de la asignatura, en dos momentos del desarrollo del curso el estudiante debe entregar el portafolios para que el contenido del mismo le sea corregido y devuelto, a fin de que introduzca las modificaciones oportunas. Igualmente en las tutorías podrán plantear las dudas 
surgidas en el proceso de confección de los trabajos, así como solicitar cuantas orientaciones consideren oportunas. El portafolios ha de incluir como entradas obligatorias los comentarios de textos, novelas, documentales y películas (Rondina, 2012), el material trabajado con respecto a las visitas, un trabajo final sobre el uso de la Edad Media en nuestros días, y una reflexión crítica de lo que ha sido su proceso de aprendizaje de la asignatura. Además de estas entradas obligatorias los alumnos podrán acordar con el profesorado otras entradas voluntarias dentro del portafolios, que tendrán una valoración positiva en la evaluación del mismo.

Para que el alumno tenga claros los criterios a partir de los cuales se van a evaluar sus trabajos, especialmente el trabajo final, se le proporciona la siguiente hoja de evaluación:

Tabla 4. Hoja de evaluación

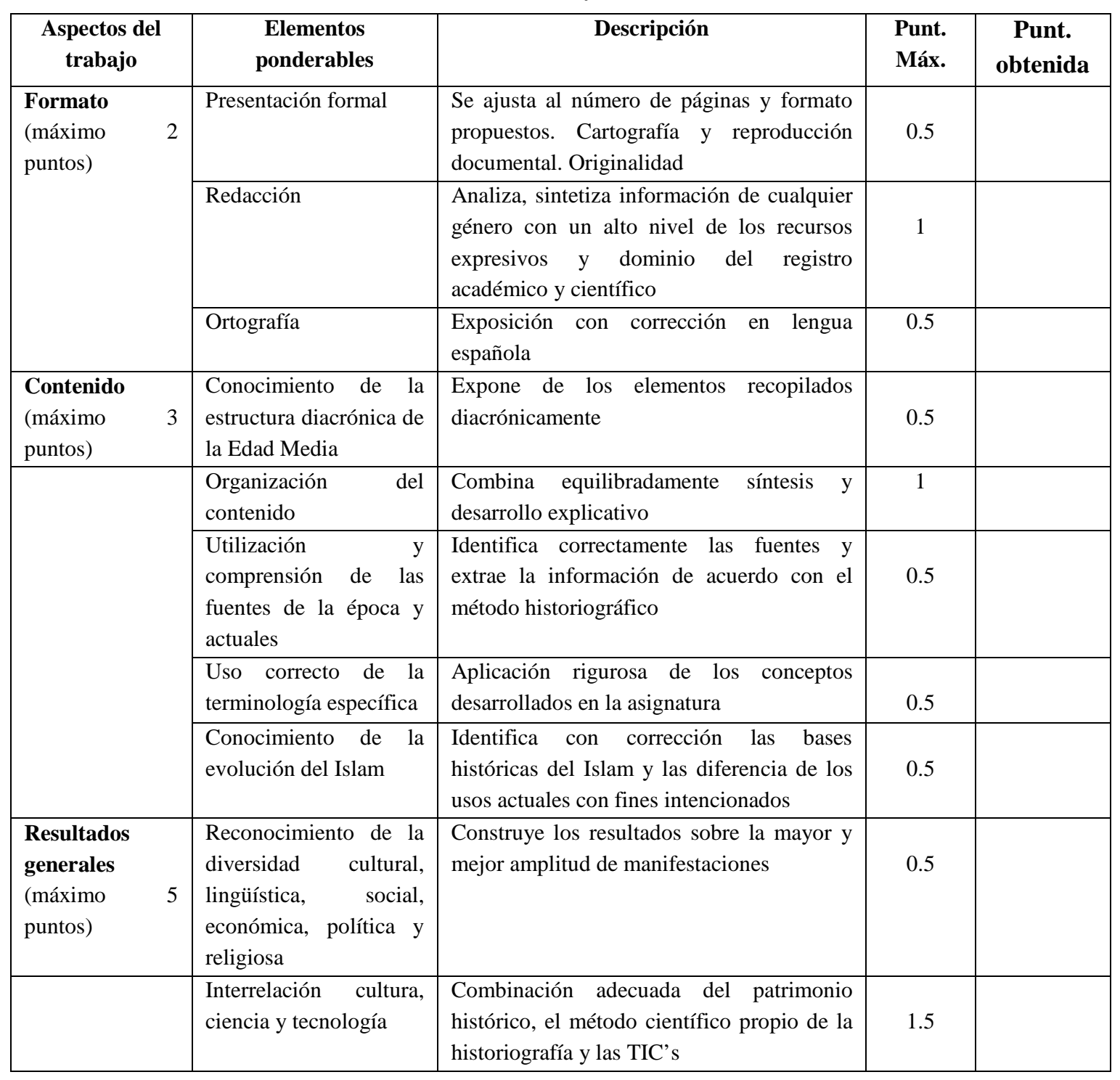




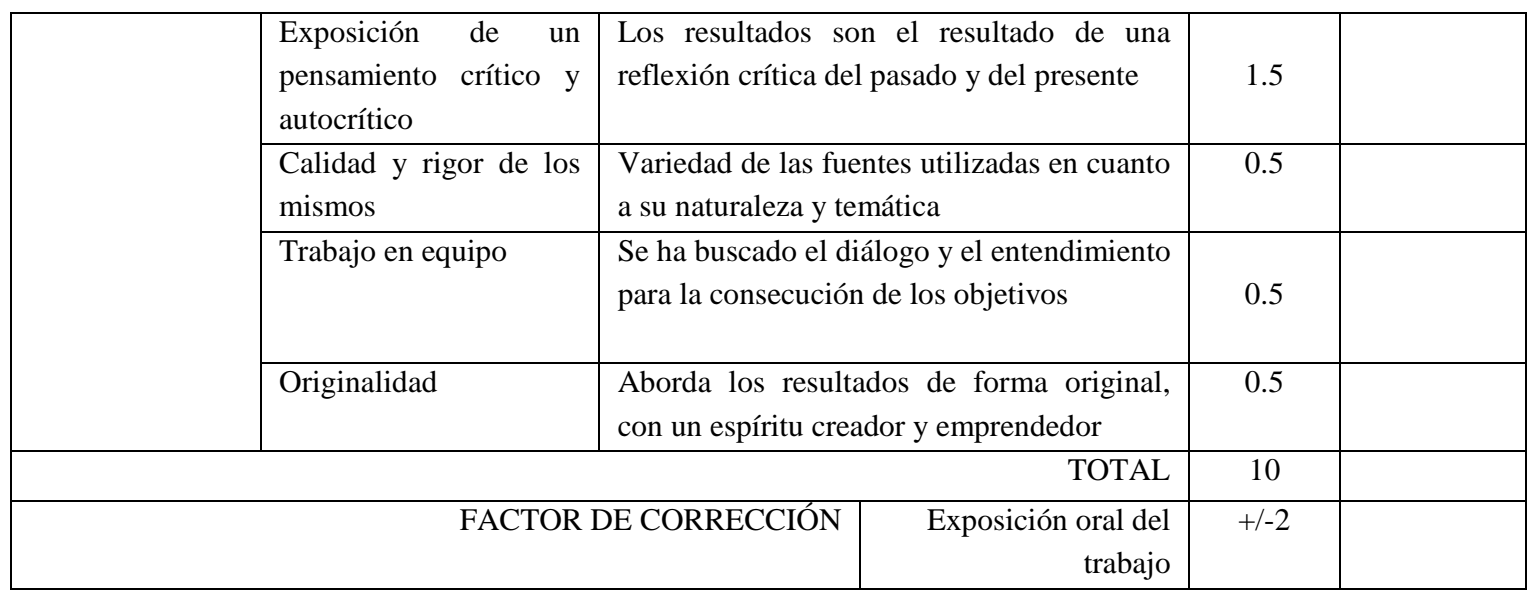

La evaluación de los contenidos teóricos de la asignatura se lleva a cabo a través de pruebas orales. A fin de facilitar la asimilación de los contenidos los estudiantes realizan las pruebas de dos en dos temas, habiendo de superar un total de dos pruebas orales (el tema 5 se evalúa a través del trabajo final). El objetivo de examinarlos oralmente no es otro que el fomentar el desarrollo de la competencia relativa a la correcta expresión oral en lengua española.

Dentro de este apartado relativo a la evaluación, las tutorías también tienen un papel importante, especialmente en lo que se refiere a la evaluación de las competencias que se van a trabajar a lo largo del curso. En el apartado sobre metodología ya hemos indicado cómo son evaluadas dichas competencias.

Finalmente, los alumnos han de participar activamente en las clases y en los debates surgidos dentro del aula y en los foros del aula virtual.

La ponderación de cada elemento a evaluar es la siguiente: portafolios: 40\%; pruebas orales: $40 \%$; tutorías: $10 \%$; foros y debates: $10 \%$.

\section{Conclusiones}

La renovación de los planes de estudio universitarios en España a raíz de la puesta en marcha de las directrices marcadas por el Espacio Europeo de Educación Superior, hace ya algunos años, supuso la necesidad de implementar proyectos de innovación docente para asignaturas nuevas que, aun siendo herederas de otras asignaturas similares en los viejos planes de estudio, necesitaban de una adaptación a la nueva realidad. Es lo que hemos presentado en este trabajo, abordando las distintas iniciativas puestas en marcha en la Universidad Pablo de Olavide para la enseñanza de la Historia Medieval.

(cc) EY-NC-ND 2018, Universitat Politècnica de València 
Desde el principio éramos conscientes del esfuerzo que habríamos de realizar para que los alumnos, acostumbrados a ser evaluados casi exclusivamente a partir de sus conocimientos teóricos, cumplieran con el proyecto aquí diseñado. Pero considerábamos que haciéndoles ver la utilidad de las herramientas y para qué servía el trabajo que iban a realizar a lo largo de la asignatura, el grado de implicación en el plan propuesto sería bastante alto, como así ha sido.

Un aspecto en el que teníamos desde el principio depositadas muchas ilusiones y confianza $^{3}$ es en el trabajo competencial. Dejando a un lado los idealismos, la inmensa mayoría de las personas que se matriculan en un título universitario lo hace con vistas a su futuro profesional. Hasta hace poco la Universidad se había limitado a ofrecerles contenidos teóricos, sin preocuparse de que los futuros profesionales llegaran a ser competentes para el mercado laboral en sus distintas actividades. En nuestro caso, nuestro objetivo no ha sido nunca conseguir egresados que sepan mucha Historia Medieval, sino que esos egresados a través de la materia que les hemos impartido y las herramientas adquiridas sean personas competentes de tal manera que el abanico de posibilidades laborales resulte lo más amplio posible.

Por último queremos resaltar que, de acuerdo con nuestra experiencia personal, el cambio siempre es absolutamente posible. La adopción de nuevos métodos de enseñanza para impartir las asignaturas, la participación más activa de los estudiantes en su proceso de aprendizaje, la mejora en los resultados académicos, el lograr egresados formados a nivel teórico y competentes, es ya una realidad. La innovación es y debe ser siempre un aspecto fundamental en nuestro proceso de formación como docentes (Romero, 2009). Un proceso donde la última etapa no existe, puesto que la innovación docente nunca debe estar concluida y cerrada.

\section{Referencias}

ALONSO CANO, C. et al. (2007). "Relatos de Innovación docente" en Revista Lationoamericana de Tecnología educativa, vol. 6, n 2, pp. 153-167.

ÁLVAREZ GONZÁLEZ, M. (2008). "La tutoría académica en el espacio europeo de la Educación Superior” en Revista interuniversitaria de formación del profesorado, $\mathrm{n}^{\circ}$ 61, pp. 7188.

BERNAL AGUDO, J.L. (2006). Pautas para el diseño de una asignatura desde la perspectiva de los ECTS. Universidad de Zaragoza. 2006.

DANVILA DEL VALLE, I. (2013). "El papel de la clase teórica en el Espacio Europeo de Educación Superior” en Estudios sobre el mensaje periodístico, $n^{\circ} 19$ (2), pp. 701-709.

\footnotetext{
3 Recuérdese la importancia de la confianza del profesor en la realización de la tarea docente es fundamental para generar actitudes positivas hacia el cambio tal como indica MARCHESI (1999)
}

(c)) EY-NC-ND 2018, Universitat Politècnica de València 
DEL VAL MERINO, C. (2013). "La integración del alumno al mundo laboral. El paradigma de la Inteligencia Emocional en el marco del Espacio Europeo de Educación Superior (EEES)” en Estudios sobre el mensaje periodístico, $\mathrm{n}^{\circ} 19$ (1), pp. 129-138.

ELBOJ, C, PUIGDELLIVOL, I, SOLER, M, VALLS, R. (2002). Comunidades de aprendizaje: transformar la educación. Barcelona: Graó.

FERNÁNDEZ ARAQUE, a. m. (2016). "La rúbrica como como instrumento de evaluación y de aprendizaje" en Formar mediante la evaluación en la Universidad: propuestas prácticas útiles para docentes. Valladolid: Universidad de Valladolid, pp. 53-64.

GIBBONS (1995). The New Production of Knowledge. London: Sage Publications.

GIL FLORES, J et al (2004). La enseñanza universitaria, planificación y desarrollo de la docencia. Madrid EOS.

HANNA, D. (2002). La enseñanza universitaria en la era digital. Barcelona: Octaedro.

KNIGHT, P. (2005). El profesorado de educación superior: formación para la excelencia. Madrid: Nancea.

LOBATÓN, g. E. (2013). La novella histórica como recurso didáctico en la enseñanza de la Historia. Tesis doctoral defendida en la Universidad Complutense de Madrid.

MARTÍN CEA, J. C., VILLANUEVA ZUBIZARRETA, O., BONACHÍA HERNANDO, J. A., DEL VAL VALDIVIESO, M. I. (2008). "Propuestas de innovación docente para el estudio de la Historia medieval “ en Innovación docente: docencia y TICs. Valladolid: Universidad de Valladolid, pp. 255-265.

MCINTOSH, C. Lifelong learning and Distance Higher Education Overview. Paris: Unesco.

MEDINA, J. L. (2002). "La deconstrucción o desaprendizaje: aproximación conceptual y notas para un método reflexivo de generación de nuevos saberes profesionales” en Revista electrónica Educare, $\mathrm{n}^{\circ} 21$ (1).

Disponible en: http://www.enfermeria21.com/revistas/educare/articulo/1021/

METCALFE, A. (2006). Knowledge management and higher education a critical analysis. London: Information Science Pub.

MIGUEL DÍAZ, M. (2006). Modalidades de enseñanza centradas en el desarrollo de las competencias. Orientaciones para promover el cambio metodológico en el marco del EEES. Oviedo: Universidad de Oviedo.

MORALES, P. (2005). Implicaciones para el profesor de una enseñanza centrada en el alumno. Madrid: Universidad Pontifica de Comillas (ICADE).

NEVOT LUNA, A. (2008). “Espacio Europeo de Educación Superior. ¿Una utopía?” en Dibujo técnico y matemáticas: una consideración interdisciplinar. Madrid: Ministerio de Educación y Ciencia, pp. 189-230.

RIVERO GRACIA, M. P. (2003). “Apuntes sobre el comentario de textos históricos” en Clío: History and History teaching, $\mathrm{n}^{\circ} 29 . \quad$ Disponible en: http://clio.rediris.es/materiales_universidad/comentario\%20texto.htm

RODRÍGUEZ DE MIÑÓN, P; BARBERO, M.I., NAVAS, M.J., SUÁREZ, J.C., HOLGADO, F.P., VILLARINO, A y RECIO, P. (2003) Recomendaciones para la elaboración de pruebas objetivas de evaluación y la interpretación de sus puntuaciones. Madrid. Facultad de Psicología de la Uned.

ROMERO MARTÍN, M. y RUESCAS NICOLAU, A. "El reto docente del Espacio Europeo de Educación Superior, ¿estamos preparados?” en Metas de enfermería, vol.12, nº9, 2009, pp. 6066.

RONDINA, G. y ANGELUCCI, M.S. (2012). “El uso del cine en la enseñanza universitaria” en Ars Boni et Aequi, vol. 8, n 1, pp. 171-201.

VELASCO MARTÍNEZ, L. (2014). "El uso de la literatura y sus adaptaciones en la enseñanza de la Historia” en Iber: Didáctica de las Ciencias Sociales, Geografía e Historia, nº 77, pp. 7380.

(cc) EY-NG-ND 2018, Universitat Politècnica de València 
Referencias electrónicas sobre webs con recursos para la enseñanza de la Historia Medieval: http://www.medievalismo.org http://labyrinth.georgetown.es http://www.tntech.edu/history/tech.html http://www.le.ac.uk/urbanhist/index.html http://www.storiadellacitta.it http://www.smr.herefordshire.gov.uk/education/medtowns_contents.html http://www.trytel.com tristan/towns/towns.html http://www.saint-denis.culture.fr/fr/index.html http://philae.sas.upenn.edu./French/caroly.html http://www.rhi.wur.nl/UK/links/rural/ http://www.arqueologiamedieval,com http://www.fordham.edu/halsall/women/womensbook.html http://www.ecohistory.net http://www.memebrs.aol.com/TeacherNet/Medieval.html http://www.library.yale.edu/rsc/history/medbib.html http://www.history-journals.de http://www.amea.unican.es http://www.journals.cambridge.org/action/display/Journal?jid=UHY http://www.edmrevistas.com/revistas/medieval http://www.jmems.dukejournals.org 